\title{
A review of safety and efficacy of zonisamide for treatment of pediatric partial epilepsy
}

This article was published in the following Dove Press journal:

Pediatric Health, Medicine and Therapeutics

14 November 2014

Number of times this article has been viewed

\section{Maria Stella Vari \\ Pasquale Striano}

Paediatric Neurology and Muscular Diseases Unit, Department of Neurosciences, Rehabilitation, Ophtalmology, Genetics, Maternal and Child Health, University of Genoa, "G Gaslini” Institute, Genova, Italy
Correspondence: Pasquale Striano Paediatric Neurology and Muscular Diseases Unit, Department of Neurosciences, Rehabilitation, Ophtalmology, Genetics, Maternal and Child Health, University of Genoa, "G Gaslini” Institute, Genova, Italy Tel +39010 5636758

Fax +390103538265

Email strianop@gmail.com
Introduction: Zonisamide is one of the most promising antiepileptic drugs that was first approved in Europe as add-on therapy in adult patients with partial seizures and recently approved as monotherapy. More recently, zonisamide has been approved for pediatric use in the UK and can now be prescribed for partial epilepsy in adolescents and children aged 6 years and above.

Aim: This paper systematically reviews the current evidence on the efficacy and tolerability of zonisamide as monotherapy and adjunctive therapy for pediatric partial epilepsy.

Methods: Relevant randomized clinical trials and open-label studies were identified by a structured PubMed search, supplemented by an additional hand search of reference lists and authors' files.

Results: PubMed database search yielded 12 (four double-blind randomized, eight open-label) clinical trials published over the last 10 years (January 2004 to September 2014) and the pooled analysis included a total of 1,555 patients treated with zonisamide.

Conclusion: Zonisamide currently represents a robust option in the treatment of children with partial epilepsy, based on its multiple mechanism of action and efficacy in different situations.

Keywords: children, clinical trial, partial seizures, tolerability

\section{Introduction}

Zonisamide is a benzisoxazole derivative chemically unrelated to other anticonvulsant agents. It shows a broad spectrum of mechanistic actions that, in part, overlap the actions of phenytoin, carbamazepine, and valproate. ${ }^{1}$ These include reduction of sustained high-frequency repetitive firing of sodium-dependent action potentials, inhibition of low-threshold T-type calcium currents, a modulatory effect on GABAmediated neuronal inhibition, inhibition of glutamate release, and weak inhibition of carbonic anhydrase. The latter may contribute to some of the side effects of this drug. Zonisamide also alters the metabolism of dopamine, 5-hydroxytryptamine, and acetylcholine. ${ }^{2,3}$ There is also evidence from in vitro studies that zonisamide may have neuroprotective properties. ${ }^{4}$

Zonisamide is completely absorbed, with a bioavailability of $100 \%$ and reaching maximum plasma concentration in 2-5 hours of oral ingestion of a dose of 200-400 $\mathrm{mg}$. This rate of absorption is slightly slower with food intake. Zonisamide is primarily excreted through the urinary tract, with a half-life of 63-69 hours. The half-life is decreased with coadministration of other isoenzyme-inducing antiepileptic drugs (AEDs), such as phenytoin, phenobarbital, carbamazepine, and sodium valproate, but still remains above 24 hours. $^{2}$ Zonisamide is metabolized by the action of isoenzyme 
3A4 in the liver, via the cytochrome P450 pathway. Since this process may be affected by other AEDs if used concomitantly, zonisamide dose adjustment may be required when used as an adjunctive treatment. ${ }^{5}$ In particular, zonisamide metabolism is affected by other AEDs, namely, hepatic enzyme inducers such as phenytoin and carbamazepine, which significantly reduce zonisamide serum levels. ${ }^{6-8}$

Zonisamide is a viable first-line and adjunctive therapy for a wide range of seizures. In particular, ZNS may be effective in generalized forms of epilepsies, featuring myoclonic and/or absence seizures, such as in patients with juvenile myoclonic epilepsy and progressive myoclonic epilepsy, although studies of these included a heterogeneous group of patients. ${ }^{9-11}$

Zonisamide is indicated in the EU as monotherapy in the treatment of partial seizures with or without secondary generalization in adults with newly diagnosed epilepsy, and as adjunctive therapy to other AEDs in the treatment of adults with partial seizures with or without secondary generalization. ${ }^{12}$ More recently, zonisamide has been approved for pediatric use in the UK and can now be prescribed for partial epilepsy in adolescents and children aged 6 years and above. ${ }^{13-16}$ Phase II studies in pediatric patients indicate that adjunctive zonisamide has an acceptable safety profile in this population and a pharmacokinetic profile similar to that observed in adult patients. ${ }^{17}$ However, there is a need for studies to determine the safety, efficacy, and tolerability of newer AEDs, such as zonisamide, in children of varying ages.

This paper systematically reviews the current evidence on the efficacy and tolerability of zonisamide as monotherapy and adjunctive therapy for the treatment of pediatric partial epilepsy.

\section{Methods}

Relevant randomized clinical trials and open-label studies in add-on or monotherapy including pediatric patients were identified by a structured PubMed search, supplemented by an additional hand search of reference lists and authors' (MSV, PS) files. Keywords for the database search included "epilepsy", "pediatric", "zonisamide", "clinical trial”, "placebo-controlled", "open-label”, and "therapy".

\section{Results}

PubMed database search yielded 12 (four double-blind randomized, eight open-label) clinical trials published over the last 10 years (January 2004 to September 2014) and the pooled analysis included a total of 1,555 patients treated with zonisamide (Table 1). In the large majority of studies, zonisamide was administered in patients with drug-resistant epilepsy, refractory to at least two AEDs.

\section{Zonisamide as adjunctive therapy}

The efficacy of zonisamide as add-on treatment in drug-resistant pediatric patients with focal seizures has been investigated in a large double-blind, placebo-controlled randomized trials in Europe and India. ${ }^{18}$ The authors recruited 207 (aged 6-17 years) patients who were taking one to two AEDs for focal seizures with or without secondary generalization. Dose adjustments were made during a titration phase, to a target dose of $8 \mathrm{mg} / \mathrm{kg} /$ day (maximum $500 \mathrm{mg} /$ day). Responder rate was assessed during the 12-week maintenance phase, and $50 \%$ of the zonisamide group had achieved a $\geq 50 \%$ reduction in partial seizure frequency, compared to $31 \%$ in the placebo group. The median decrease in seizure frequency from baseline was $50 \%$ for zonisamide, compared to $24.5 \%$ for placebo, a difference that reached statistical significance. The authors concluded that adjunctive zonisamide treatment was shown to be effective and well tolerated in pediatric patients with partial epilepsy. There were three open-label studies involved mixed cohorts of both children and young adults. ${ }^{19-21}$ Coppola et $\mathrm{al}^{20}$ reported seizure freedom in $10.9 \%$ of their sample, and a responder rate in $37.8 \%$, at a mean dose of $5.7 \mathrm{mg} / \mathrm{kg} /$ day as an add-on therapy in refractory partial epilepsy. Kluger et $\mathrm{al}^{19}$ assessed the use of zonisamide in 24 patients with partial epilepsy. Responder rates were $58.3 \%$ at 8 weeks and $41.7 \%$ at 18 months. Guerrini et al's ${ }^{21}$ extension study reported seizure freedom in $16 \%$ and a responder rate in $56.3 \%$ at dose of $8 \mathrm{mg} / \mathrm{kg} /$ day as an add-on therapy in refractory epilepsy. Another study looking at the use of zonisamide as an adjunctive therapy in pediatric populations reported a median decrease in seizure frequency of $1.3 \%$ per week, which approached statistical significance $(P=0.056)$. Responder rate was not reported in this study. ${ }^{22}$

Furthermore, a retrospective case-notes review of pediatric patients treated with adjunctive zonisamide found a low responder rate of $23.5 \%$ whereas the incidence of adverse effects was lower than other studies. ${ }^{23}$ Two retrospective studies were undertaken in patients with intractable epilepsy. ${ }^{24,25}$ In the first study, 35 children (age: 8 months to 22 years; mean: 9 years) showed good to excellent seizure control (50\%-100\% reduction) in $42.8 \%$ of the all treated patients. Adverse effects were not associated with a higher mean dose. In the second retrospective study, $48.5 \%$ of 163 patients experienced a reduction in seizure frequency of more than 


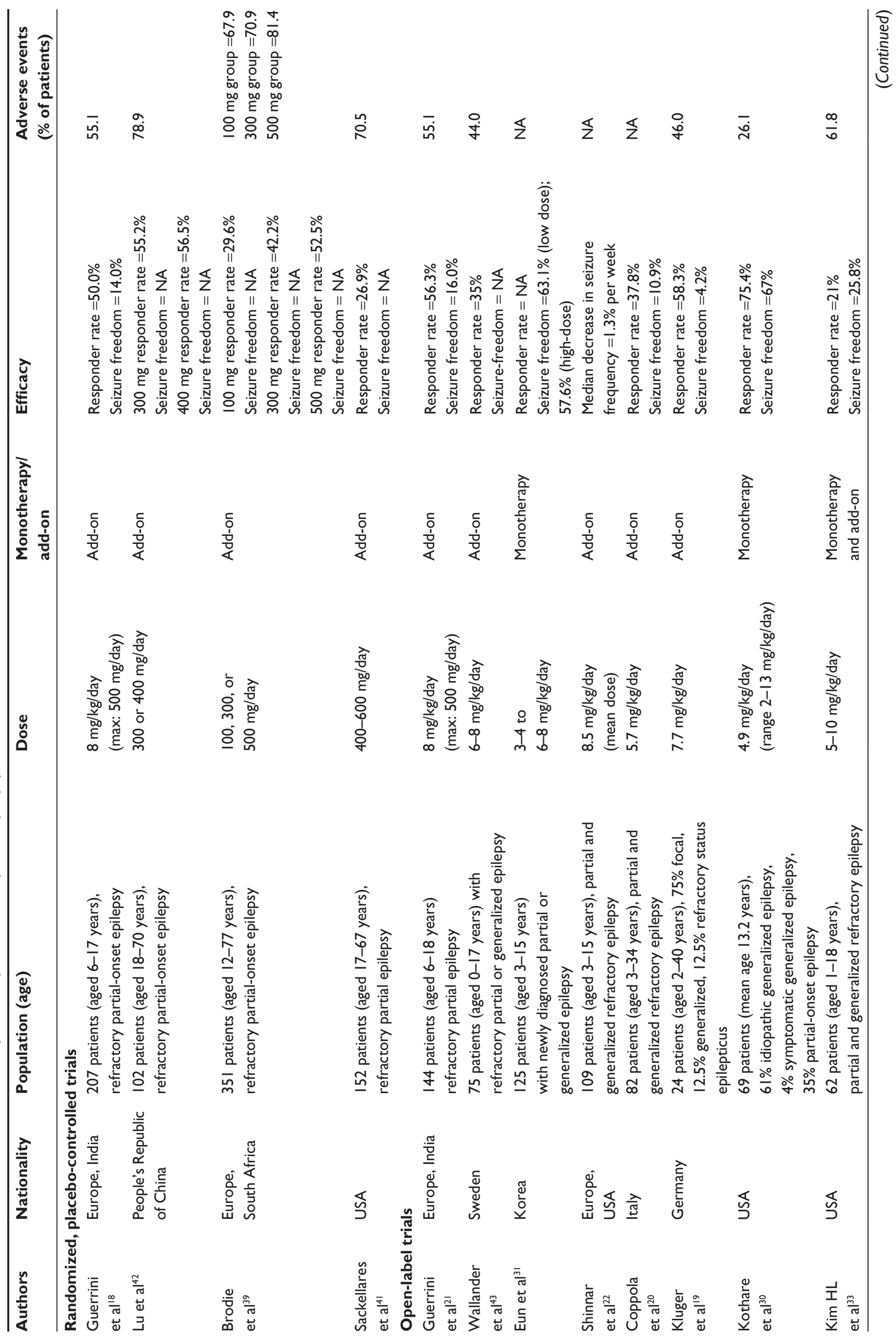




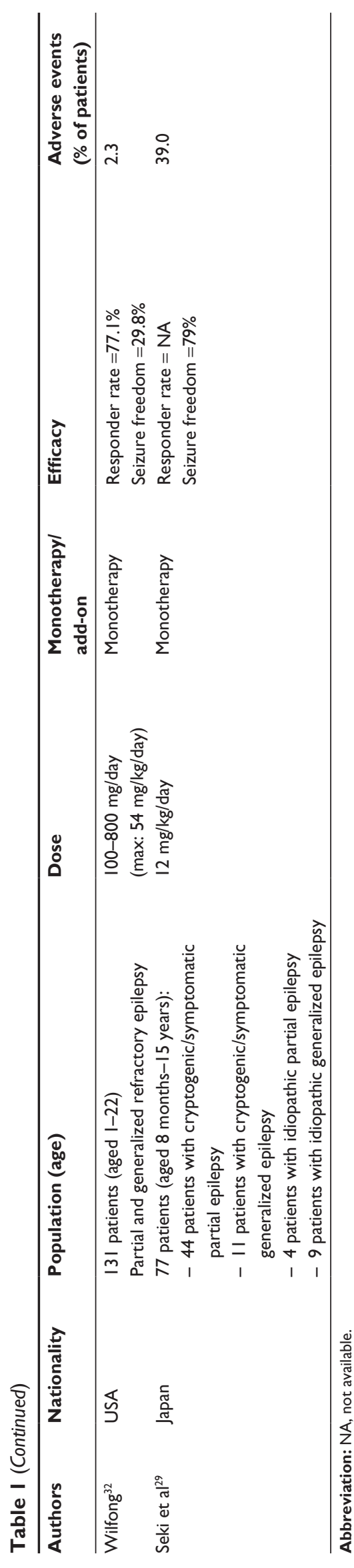

$50 \%$ after ZNS adjunctive therapy, with 25 patients (15.3\%) being seizure-free.

\section{Zonisamide as monotherapy}

There have been multiple retrospective chart-review studies suggesting the potential efficacy and safety of zonisamide as monotherapy in refractory partial seizures in adults and across the lifespan. ${ }^{26-28}$ Overall, the evidence to support the use of zonisamide in pediatric populations, particularly as monotherapy, is still limited. The available studies are summarized in Table 1.

Three open-label studies considered the use of zonisamide as monotherapy in children. ${ }^{29-31}$ In these studies, seizure freedom ranged from $57 \%$ to $79 \%$ of patients; no significant differences in seizure-freedom rates were found between low- or high-dose zonisamide. Another open-label trial, including both pediatric and young adult population (aged 1-22 years), reported a lower rate of seizure freedom $(29.8 \%)$ whereas a total of 101 patients $(77.1 \%)$ achieved a $50 \%$ or greater decrease in seizure frequency (ie, responders). ${ }^{32}$

An additional two open-label studies evaluated the efficacy of zonisamide in children with epilepsy, but did not report separated results for patients on monotherapy or polytherapy. ${ }^{33,34}$

The large variability among the rate of seizure-free patients between the different trials can be explained by different factors, including their open-label nature and, mostly, the recruitment of adult patients in some studies.

In summary, the available literature suggests that zonisamide can be useful as monotherapy in pediatric patients who had received no previous treatment and in newly diagnosed partial epilepsy. However, further evaluation is needed with respect to efficacy, safety, and effectiveness in this population.

\section{Safety and tolerability data}

The pharmacokinetic profile of zonisamide has a number of advantages. First, the long half-life allows once-daily dosing, improves patient compliance, and suggests a low impact of missed doses on seizure control. However, it has to be acknowledged that the long half-life may represent a limitation when a rapid onset of action is required. In general, the majority of the reviewed trials suggest that zonisamide is an effective and well tolerated treatment option for pediatric patients with focal epilepsy. The occurrence of side effects in the different trials ranged from $26 \%$ to $81.4 \% .{ }^{18-20}$ Side effects were usually mild in severity and resolved shortly after 
discontinuation of the drug. The most frequently reported side effects included decreased appetite, decreased weight, somnolence, nausea/vomiting, and diarrhea; additionally, both psychiatric (eg, psychosis and suicidal ideation) or cognitive adverse effects were reported, and the high-dose group was significantly worse than that in the low-dose group, but that is in line with other new-generation AEDs. ${ }^{29,35}$ In many studies, patients experienced side effects during the escalation phase, of which the majority resolved during the maintenance of the medication. ${ }^{30}$

Teratogenic effects of zonisamide were not clearly defined from these studies even if the present data do not indicate that the risk of teratogenicity is greater than that of other conventional AEDs. ${ }^{36}$ However, such risk cannot be neglected even at therapeutic dosages or concentrations of ZNS, especially in patients receiving polytherapy. In addition, postmarketing studies conducted in Japan revealed that pediatric patients on zonisamide have an increased risk for oligohydrosis and hyperthermia. Therefore, it is advisable that patients would be monitored closely for signs of decreased sweating and increased body temperature, especially in warm or hot weather. $^{37}$

\section{Discussion}

Zonisamide (3-sulfamoylmethyl-1, 2-benzisoxazole) is an AED with a broad spectrum of mechanistic action that has demonstrated good efficacy in controlling seizures as an add-on therapy in adult and pediatric epilepsy. ${ }^{25,38-40}$ Despite some methodological drawbacks, our review of available literature supports the view that adjunctive zonisamide therapy is effective and well tolerated in pediatric patients with refractory partial epilepsy, and could therefore prove to be a useful new treatment option for this patient population. The evidence in support of the use of zonisamide in pediatric populations, as monotherapy, is still limited, indicating the need for further investigation in randomized, controlled trials. In the available clinical trials, the titration schedule of the drug varied but, in general, the recommended dosage escalation starts at $1 \mathrm{mg} / \mathrm{kg} /$ day and increases at weekly intervals in increments of $1 \mathrm{mg} / \mathrm{kg}$ to the target dose of 6-8 $\mathrm{mg} / \mathrm{kg} /$ day up to a maximum dose of 300-500 mg/day in two daily doses. Of note, Sackellares et al showed that a slower (or 2-weekly) titration of zonisamide was associated with fewer adverse effects. ${ }^{41}$

The tolerability profile of zonisamide was high in the large majority of the studies, both in add-on and monotherapy. From the review of the literature (Table 1), the risk of significant side effects is higher in patients in polytherapy.
Notably, there are very limited data from clinical studies in patients with a body weight of less than $20 \mathrm{~kg}$. Therefore, children aged 6 years and above and with a body weight less than $20 \mathrm{~kg}$ should be treated with caution.

In conclusion, zonisamide, as adjunctive therapy, and potentially as monotherapy, is successful in treating pediatric patients with refractory partial seizures because of its structural and mechanistic profile, safety, tolerability, and wide spectrum of antiseizure activity. The challenge for new more-efficacious, more-specific, and better-tolerated drugs is also continuing. However, the ultimate goal should be to not only render the patients seizure-free, but also to allow for improvement of quality of life of individuals and to reduce the costs of medical care.

\section{Disclosure}

The authors report no conflict of interest in this work.

\section{References}

1. Peters DH, Sorkin EM. Zonisamide. A review of its pharmacodynamic and pharmacokinetic properties, and therapeutic potential in epilepsy. Drugs. 1993;45(5):760-787.

2. Kochak GM, Page JG, Buchanan RA, Peters R, Padgett CS Steady-state pharmacokinetics of zonisamide, an antiepileptic agent for treatment of refractory complex partial seizures. J Clin Pharmacol. 1998;38(2):166-171.

3. Afra P, Adamolekun B. Update on once-daily zonisamide monotherapy in partial seizures. Neuropsychiatr Dis Treat. 2014;10:493-498.

4. Rösler TW, Arias-Carrión O, Höglinger GU. Zonisamide: aspects in neuroprotection. Exp Neurol. 2010;224(2):336-339.

5. Zonegran ${ }^{\circledR}$ (zonisamide) capsules [package insert]. Woodcliff Lake, NJ: Eisai Pharmaceuticals Inc.; 2006.

6. Minami T, Ieiri I, Ohtsubo K, et al. Influence of additional therapy with zonisamide (Excegran) on protein binding and metabolism of carbamazepine. Epilepsia. 1994;35(5):1023-1025.

7. Tasaki K, Minami T, Ieiri I, et al. Drug interactions of zonisamide with phenytoin and sodium valproate: serum concentrations and protein binding. Brain Dev. 1995;17(3):182-185.

8. Ojemann LM, Shastri RA, Wilensky AJ, et al. Comparative pharmacokinetics of zonisamide (CI-912) in epileptic patients on carbamazepine or phenytoin monotherapy. Ther Drug Monit. 1986;8(3):293-296.

9. Italiano D, Pezzella M, Coppola A, et al. A pilot open-label trial of zonisamide in Unverricht-Lundborg disease. Mov Disord. 2011;26(2):341-343.

10. Striano P, Belcastro V. Treating myoclonic epilepsy in children: state-of-the-art. Expert Opin Pharmacother. 2013;14(10):1355-1361.

11. Zaccara G, Specchio LM. Long-term safety and effectiveness of zonisamide in the treatment of epilepsy: a review of the literature. Neuropsychiatr Dis Treat. 2009;5:249-259.

12. Hoy SM. Zonisamide: a review of its use in the management of adults with partial seizures. Drugs. 2013;73(12):1321-1338.

13. Leppik IE. Three new drugs for epilepsy: levetiracetam, oxcarbazepine, and zonisamide. J Child Neurol. 2002;17 Suppl 1:S53-S57.

14. Schulze-Bonhage A. Zonisamide in the treatment of epilepsy. Expert Opin Pharmacother. 2010;11(1):115-126.

15. Kito M, Maehara M, Watanabe K. Mechanisms of T-type calcium channel blockade by zonisamide. Seizure. 1996;5(2):115-119.

16. Mula M. Profile of once-daily zonisamide as monotherapy for treatment of partial seizures in adults. Drug Des Dev Ther. 2013;7:397-402. 
17. Brodie MJ, Ben-Menachem E, Chouette I, Giorgi L. Zonisamide: its pharmacology, efficacy and safety in clinical trials. Acta Neurol Scand Suppl. 2012;(194):19-28.

18. Guerrini R, Rosati A, Segieth, J, Pellacani S, Bradshaw K, Giorgi L. A randomized phase III trial of adjunctive zonisamide in pediatric patients with partial epilepsy. Epilepsia. 2013;54(8):1473-1480.

19. Kluger G, Zsoter, A, Holthausen H. Long-term use of zonisamide in refractory childhood-onset epilepsy. Eur J Paediatr Neurol. 2008;12(1):19-23.

20. Coppola G, Grosso S, Verrotti A, et al. Zonisamide in children and young adults with refractory epilepsy: an open label, multicenter Italian study. Epilepsy Res. 2009;83(2-3):112-116.

21. Guerrini R, Rosati A, Bradshaw K, Giorgi L. Adjunctive zonisamide therapy in the long-term treatment of children with partial epilepsy: results of an extension study of a phase III, randomized, double bind, placebo-controlled trial. Epilepsia. 2014;55(4):568-578.

22. Shinnar S, Pellock JM, Conry JA. Open-label, long-term safety study of zonisamide administered to children and adolescents with epilepsy. Eur J Paediatr Neurol. 2009;13(1):3-9.

23. Tan HJ, Martland TR, Appleton RE, Kneen R. Effectiveness and tolerability of zonisamide in children with epilepsy: a retrospective review. Seizure. 2010;19(1):31-35.

24. Mandelbaum DE, Bunch M, Kugler SL, Venkatasubramanian A, Wollack JB. Broad-spectrum efficacy of zonisamide at 12 months in children with intractable epilepsy. J Child Neurol. 2005;20(7):594-597.

25. Lee YJ, Kang HC, Seo JH, Lee JS, Kim HD. Efficacy and tolerability of adjunctive therapy with zonisamide in childhood intractable epilepsy. Brain Dev. 2010;32(3):208-212.

26. Faught E. Review of United States and European clinical trials of zonisamide in the treatment of refractory partial-onset seizures. Seizure. 2004;13 Suppl 1:S59-S65; discussion S71-S72.

27. Villanueva V, Serrano-Castro PJ. [Zonisamide in the epilepsy treatment: a literature review from add-on therapy to monotherapy]. Rev Neurol. 2013;56(8):429-438. Spanish.

28. Cox JH, Seri S, Cavanna AE. Zonisamide as a treatment for partial epileptic seizures: a Systematic review. Adv Ther. 2014;31(3):276-288.

29. Seki T, Kumagai N, Maezawa M. Effects of zonisamide monotherapy in children with epilepsy. Seizure. 2004;13 Suppl 1:26-32; discussion S33.

30. Kothare SV, Kaleyias J, Mostofi N, et al. Efficacy and safety of zonisamide monotherapy in a cohort of children with epilepsy. Pediatr Neurol. 2006;34(5):351-354.
31. Eun SH, Kim HD, Eun BL, et al. Comparative trial of low- and highdose zonisamide as monotherapy for childhood epilepsy. Seizure. 2011;20(7):558-563.

32. Wilfong AA. Zonisamide monotherapy for epilepsy in children and young adults. Pediatr Neurol. 2005;32(2):77-80.

33. Kim HL, Aldridge J, Rho JM. Clinical experience with zonisamide monotherapy and adjunctive therapy in children with epilepsy at a tertiary care referral center. J Child Neurol. 2005;20(3):212-219.

34. Iinuma K, Haginoya K. Clinical efficacy of zonisamide in childhood epilepsy after long-term treatment: a postmarketing, multi-institutional survey. Seizure. 2004;13 Suppl 1:S34-S39; discussion S40.

35. Cavanna AE, Seri S. Psychiatric adverse effects of zonisamide in patients with epilepsy and mental disorders comorbidities. Epilepsy Behav. 2013;29(2):281-284.

36. Kondo T, Kaneko S, Amano Y, Egawa I. Preliminary report on teratogenic effects of zonisamide in the offspring of treated women with epilepsy. Epilepsia. 1996;37(12):1242-1244.

37. Wilfong AA, Willmore LJ. Zonisamide - a review of experience and use in partial seizures. Neuropsychiatr Dis Treat. 2006;2(3):269-280.

38. Marinas A, Villanueva V, Giràldez BG, Molins A, Salas-Puig J, Serratosa JM. Efficacy and tolerability of zonisamide in idiopathic generalized epilepsy. Epileptic Disord. 2009;11(1):61-66.

39. Brodie MJ, Duncan R, Vespignani H, Solyom A, Bitenskyy V, Lucas C. Dose-dependent safety and efficacy of zonisamide: a randomized, double-blind, placebo-controlled study in patients with refractory partial seizures. Epilepsia. 2005;46(1):31-41.

40. You SJ, Kang HC, Kim HD, Lee HS, Ko TS. Clinical efficacy of zonisamide in Lennox-Gastaut syndrome: Korean multicentric experience. Brain Dev. 2008;30(4):287-290.

41. Sackellares JC, Ramsay RE, Wilder BJ, Browne TR 3rd, Shellenberger MK. Randomized, controlled clinical trial of zonisamide as adjuctive treatment for refractory partial seizures. Epilepsia. 2004; 45(6):610-617.

42. Lu Y, Xiao Z, Yu W, Xiao F, Xiao Z, Hu Y, Chen Y, Wang X. Efficacy and safety of adjunctive zonisamide in adult patients with refractory partial-onset epilepsy: a randomized, double-blind, placebo-controlled trial. Clin Drug Investig. 2011;31:221-229.

43. Wallander KM, Ohman I, Zonisamide DM. Pharmacokinetics, Efficacy, and Adverse Events in Children with Epilepsy. Neuropediatrics. Epub 2014 Aug 18.
Pediatric Health, Medicine and Therapeutics

\section{Publish your work in this journal}

Pediatric Health, Medicine and Therapeutics is an international, peerreviewed, open access journal publishing original research, reports, editorials, reviews and commentaries. All aspects of health maintenance, preventative measures and disease treatment interventions are addressed within the journal. Practitioners from all disciplines are invited to submit

\section{Dovepress}

their work as well as healthcare researchers and patient support groups. The manuscript management system is completely online and includes a very quick and fair peer-review system. Visit http://www.dovepress.com/ testimonials.php to read real quotes from published authors. 